\title{
Impacto de los proyectos universitarios en el desarrollo local del municipio Camajuaní, Cuba
}

\author{
Impact of university projects on the local development of Camajuaní municipality, Cuba
}

\author{
Alexander Báez Hernández ${ }^{1}$, Carlos Alberto Hernández \\ Medina $^{2}$, Magdalys Alibet Carrasco Fuentes ${ }^{3}$ \\ ${ }^{1}$ Universidad Central de Ecuador. albaez@uce.edu.ec. \\ ${ }^{2}$ Universidad Central Marta Abreu de Las Villas. cahm862@uclv.edu.cu \\ ${ }^{3}$ Universidad Central “Marta Abreu”de Las Villas. alibet7305@uclv.edu.cu
}

\section{OPEN ACCESS}

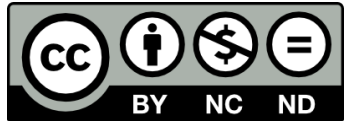

Copyright:(c) 2019 Ingenierías USBmed. La revista Ingenierías USBmed proporciona acceso abierto a todos sus contenidos bajo los términos de la licencia creative commons Atribución- no comercial- SinDerivar 4.0 Internacional (CC BY-NC-ND 4.0)

Tipo de Artículo: Investigación cientifica y tecnológica

Recibido:25-09-2018.

Revisado:.08-01-2019.

Aprobado: 11-02-2019.

Doi: $10.21500 / 20275846.3746$

Referenciar así: A.,Báez-Hernandez., C.M., Hernandez-Medina., and M., Carrasco- Fuentes. "Impacto de los proyectos universitarios en el desarrollo local del municipio Camajuaní, Cuba”. Ingenierías USBMed, 10(1), pp.19-27, 2019.

Declaración de disponibilidad de datos: Todos los datos relevantes están dentro del artículo, así como los archivos de soporte de información.

Conflicto de intereses: los autores han declarado que no existen conflicto de intereses.

Editor: Yohana López Rivera, Universidad de San Buenaventura, Medellín, Colombia
Resumen. El trabajo explora cómo contribuyen los proyectos al fortalecimiento del Desarrollo Agropecuario Local de un municipio cubano. Se precisan los aportes de la gestión de proyectos al tejido productivo en las cooperativas campesinas desde la investigación de profesores y estudiantes. Los proyectos juegan un papel importante en el fortalecimiento del tejido productivo en Camajuaní y contribuyen al Desarrollo Local en la generación de empleos, incorporación de la mujer al trabajo agrícola, incrementando la repoblación forestal, incorporando nuevas variedades de bambú que pueden ser utilizadas en obras constructivas y fabricación de muebles y útiles del hogar, ampliado infraestructuras y mejorando las existentes, diversificando la producción y produciendo abonos naturales y materia orgánica para lograr mayor fertilidad del suelo y productos más saludables.

Palabras Clave. Alimentos, Cooperativas, Impactos, Proyectos, Sostenibilidad.

Abstract. The paper explores how contribute the projects to invigoration of Local Agricultural Development of a Cuban municipality. It is necessary the contribution from projects managenment to productive network in rural cooperatives from the investigation of professors and students. Projects play an important paper in invigoration of productive network in Camajuaní and they contribute to the Local Development in generation of employments, incorporation of woman to agricultural work, increasing forest repopulation, incorporating new bamboo varieties that can be used in constructive works and production of furniture and useful of home, enlarged infrastructures and improving the existent ones, diversifying production and producing natural payments and organic matter to achieve bigger fertility of soil and healthier products.

Keywords. Projects, Foods, Cooperative, Impacts, Sustainability. 


\section{Introducción}

A. Los proyectos como dinamizadores del tejido productivo local.

Haciendo un análisis del término proyecto puede plantearse que este suele utilizarse en contextos muy variados y con significados diversos. Así, se puede encontrar que en algunos casos se hace referencia a un proyecto como "una idea más o menos articulada y planificada de cómo dar respuesta a un problema o necesidad" [1]. Es, por tanto, una propuesta a desarrollar posteriormente o que está ya en proceso de desarrollo. Se entiende por proyecto, "el plan coherente dirigido a cumplir con determinado objetivo no rutinario, se ejecuta una sola vez y tiene una fecha de duración finita, con una fecha de inicio y otra de terminación esperada" [2]. Se puede observar de una manera mas amplia como "la combinación de recursos humanos y no humanos, en una organización temporal y con un propósito determinado" [3]. Koch[4], al referirse a un proyecto plantea: "proyectar es para mí una pasión, pero más importante es sentirse parte de un sueño, y de algo tangible de algo que tú hiciste posible".

\section{B. Los proyectos y el fortalecimiento del tejido productivo}

Los proyectos, orientados a dar respuesta a las demandas de los actores individuales y sociales de base, resultan de vital importancia para promover el desarrollo humano local [5]. No tienen éxito si no generan procesos de participación, cooperación, implicación entre los actores y sus familiares no se involucran en la búsqueda de soluciones viables a los problemas en la esfera de la producción, medioambientales, que tienen que ver con la formación agroecológica y el mejoramiento de la situación económica y social de las familias campesinas del territorio [6].

La intervención mediante proyectos debe promover la movilización de los individuos que viven y laboran en los territorios rurales y formar capacidades, conocimientos y habilidades para gestar el desarrollo en cada uno de los espacios donde desenvuelven sus actividades productivas, sociales, y culturales [7]. Todos los proyectos orientan sus acciones a una mejor combinación de los factores de la producción y otros recursos locales [8]. Así generan nuevas formas organizacionales y de cooperación en las actividades productivas y socio-comunitarias de los miembros de las familias campesinas [9].

También promueve acciones relativas a actividades económicas como el suministro de insumos, promoción de nuevas formas de cooperación productiva que involucra a otros productores, pobladores y actores locales, el apoyo de diferentes instituciones y la presencia de la mujer [10]. Tributa al objetivo de aumentar la producción por la vía del incremento los rendimientos. Promueve la reconstrucción de las relaciones productivas desde la base en torno a interese y objetivos comunes [11].

Los municipios constituyen en Cuba lo que se denomina como espacio local y es allí donde radica la raíz de la estrategia de desarrollo. Por ello cada municipio trabaja sus potencialidades y barreras para, en base a ello, trazar las líneas estratégicas que le permitan gestionar sus propios proyectos o incorporarles elementos locales a proyectos de origen nacional que se destinen a la localidad[12] .

Camajuaní, municipio de la provincia de Villa Clara, no está exento de esta estrategia de desarrollo. Camajuaní cuenta con potencialidades que se analizaron para la posterior formulación de proyectos que se sustentan en ellas y que puedan contribuir al fortalecimiento del tejido productivo y todas las relaciones de cooperación que puedan surgir en dicho entramado. Entre ellas destacan: un pueblo laborioso, preparado y comprometido con el municipio; personas con un nivel de creatividad e innovación a explotar; tierras fértiles y campesinos con conocimientos y experiencia, más de 4000 productores vinculados directamente a la producción; infraestructura y tradiciones de producción agropecuaria en la localidad con personal experimentado [8].

Se debe potenciar la integración pertinente entre las universidades y los Centros Universitarios Municipales y el uso de estudiantes del municipio que estudian en las universidades en proyectos de producción de alimentos, vivienda o TIC para el desarrollo local [13]. El Objetivo General del trabajo es explorar como contribuyen los proyectos al fortalecimiento del tejido productivo del sector agrícola y al desarrollo agropecuario local del municipio Camajuaní.

\section{Desarrollo del artículo}

\section{A. Metodología}

Para el logro del objetivo planteado en la investigación se utilizaron diversos métodos. Del nivel empírico usamos la revisión de documentos, entrevistas no estructuradas al coordinador de los proyectos en el municipio y representantes de diversas instituciones que pudieran ofrecer información sobre la ejecución de los proyectos y estudio de casos. Del nivel teórico usamos: el método analítico-sintético, el lógicohistórico y el inductivo-deductivo. Los métodos y técnicas utilizados en la investigación son: la revisión de documentos facilitados por las instituciones comprometidas con el proceso de desarrollo local y la vida económica y productiva del territorio como: la Asamblea Municipal del Poder Popular, la Dirección Municipal de Economía y Planificación, la Delegación Municipal de la Agricultura, la Asociación Nacional de Agricultores Pequeños, el Museo y el Centro Universitario Municipal. También se utilizó la entrevista no estructurada para corroborar y actualizar la información recogida a partir de los documentos estudiados. Por último se realizó el estudio de casos para constatar el papel y significado de la introducción de proyectos en el sector de la agricultura y en la actividad productiva del municipio para fortalecer el tejido productivo.

Además de los documentos mencionados con anterioridad; para profundizar en algunos aspectos se hizo necesario revisar la Ley No. 95, Ley de Cooperativas de Producción Agropecuaria y de Créditos y Servicios, el Informe de Mo- 
vimientos Productivos elaborado por la ANAP el 4 de junio de 2016 y el Balance de Áreas del MINAGRI. Para precisar algunos datos y conformar todo el entramado productivo fueron entrevistados funcionarios como el miembro del Buró Municipal de la ANAP, el delegado de funcionamiento y desarrollo, el director de la Granja Urbana, etc.

Tras la revisión de documentos como el Diagnóstico de los Escenarios Municipales elaborado por la Dirección Municipal de Economía y Planificación de Camajuaní en enero del 2016, el Registro de Entidades Económicas del 2015 también elaborado por la misma entidad, el Plan de Autoabastecimiento Municipal del 2016 y otros se pudo obtener un tejido productivo local bastante acertado del municipio, haciendo énfasis en el sector agrícola.

\section{B. Caracterización del municipio de Camajuaní}

El municipio se encuentra situado al norte de la provincia de Villa Clara, con 7 límites geográficos, al norte con el Océano Atlántico, al este con Caibarién y Remedios, al oeste con Santa Clara, Cifuentes y Encrucijada y al Sur con Santa Clara y Placetas. Cuenta con una extensión territorial de $612.88 \mathrm{~km} 2$, quinto municipio de la provincia en ese indicador con el 7\% del territorio provincial. Se caracteriza por una topografía con relieve de llanura con rasgos fluviomarinos deltaicos y pantanosos, en un paisaje erosionado y diseccionado con algunas alturas residuales. Las lomas de Santa Fe constituyen la mayor altura del municipio con más de 200 metros sobre el nivel del mar. La superficie total es de 61287.88 ha., de ellas 50310.60 ha. son de superficie agrícola. 33427.83 ha. son cultivadas y el $85.5 \%$ de la superficie agrícola está en el sector no estatal[14].

Cuenta con una población de 62429 habitantes, con una tendencia al decrecimiento del $0.8 \%$ en los últimos años. Igualmente sucede con la estructura rural y urbana con un crecimiento significativo del grado de urbanización que ya alcanza el 59\% creciendo en los últimos años más de un $10 \%$. En edad laboral existen en el municipio alrededor de 36289 personas, de ellos, ocupados en la actividad estatal 11 801 y en la no estatal 10728 . Las 13461 personas restantes se ocupan en la economía informal lo que incluye las amas de casas. Existen 299 desocupados.

La población del territorio esta concentrada en 46 asentamientos donde reside el $88 \%$ del total y un $12 \%$ es disperso, todos ellos en los 13 Consejos Populares y 126 Circunscripciones. De los 13 Consejos Populares son urbanos, Camajuaní I y II, Vueltas, La Quinta y Vega Alta.

El tejido productivo del territorio del municipio se sustenta en una infraestructura física que se caracteriza por poseer: importantes recursos hidráulicos como el río Camajuaní que atraviesa el territorio de Sur a Norte, como afluente del Sagua la Chica, con importante caudal. Además, posee la presa La Quinta con capacidad disponible de 32 millones de $m^{3}$ de agua. También posee 35 microembalses con una capacidad de 0.9 millones de $m^{3}$ de agua.
El municipio se estructura en 13 Consejos Populares, 3 Urbanos y 10 rurales y su principal renglón económico es la agricultura basada en 6 objetivos productivos: Cultivos Varios, Horticultura, Tabaco, Caña de Azúcar, Arroz y Ganadería, ya que cuenta con suelos fértiles y profundos.

Se cuenta con un área agrícola de 49456.86 ha., que representa el $82.0 \%$ del territorio, con el $66.4 \%$ de superficie cultivada. De ella el $31.0 \%$ es de caña de azúcar y el $35,4 \%$ de otros cultivos. Solo el 6,4\% de la superficie agrícola está declarada como tierra ociosa. Hay entre tierras ociosas y deficientemente explotadas un total de 2018.869 ha. de las cuales están en proceso de entrega 139.22 ha. que se destinarán a 6 líneas fundamentales de producción: cultivos varios, tabaco, frutales, arroz, ganadería y ganado menor. Existe un área bajo riego de 46301.35 ha.

La Granja Urbana es responsable de implementar 29 subprogramas de la agricultura con el objetivo de lograr la aplicación científico-técnica vigente en cada uno. Dentro de sus producciones principales están las hortalizas y las uvas para lo cual cuentan con 6 organopónicos, 2 huertos intensivos, 2 fincas de frutales y una finca de semillas. Hasta la fecha tienen producido entre viandas y hortalizas 136 toneladas por año las cuales se destinan para el consumo social a organismos priorizados y para la venta directa a la población.

El Centro de Reproducción de Entomófagos y Entomopatógenos fabrica biopreparados de origen microbiano para el control de plagas agrícolas y enfermedades en diferentes cultivos de cítricos, arroz, maíz, boniato, tabaco, plátano, leguminosas, gramíneas y hortalizas. Mensualmente produce $80 \mathrm{~kg}$. de Beauveria bassiana y $385 \mathrm{~kg}$. de Bacillus thuringiensis y tiene un potencial productivo de $1000 \mathrm{~kg}$. mensuales.

La Granja Estatal Batalla de Santa Clara cuenta con una superficie agrícola de 834.46 ha. y una no agrícola de 60.92 ha., ociosa 99.81 hectáreas. La Pecuaria Luís Arcos Bergnes de Vueltas se dedica fundamentalmente a la ceba de toros. Tiene una superficie agrícola de 2935.70 ha., de estas, dedicadas a pastos y forrajes 491.84 y ociosas 804.40 ha.

El establecimiento de Tabaco "La Estrella" cuenta con una dependencia de cultivos varios que dedica sus producciones al autoconsumo en comedores. Además cuenta con 9 escogidas a donde se destina el tabaco comprado para ser beneficiado. Este establecimiento compra las producciones de los campesinos en su totalidad y es encargado de suministrarle todo lo necesario para su cosecha con calidad. El $49 \%$ del tabaco que se compra es para la exportación.

El sector cooperativo y campesino es el sector de mayor peso en la agricultura del municipio. Cuenta con 45 organizaciones de base que son 13 CPA y 32 CCS. Estas agrupan a 4875 socios de los que 4385 son hombres y 490 mujeres. Las cooperativas cuentan con 568.70 cab. de tierra (7631.95 ha.) en CPA y 1657.12 cab. (22 870.24 ha.) en CCS, para un total de 2225.80 cab. de tierras (29 870.24 ha) . 
C. Papel de los proyectos en el fortalecimiento del tejido productivo agrícola de Camajuaní

En el municipio se están llevando a cabo por parte de la ANAP, CUM y ACTAF los proyectos:

Fomento de la producción de Palma Real para la reforestación en la CCS-F "Arnaldo Arteaga".

Proyecto Post-Cosecha: "Fabricación de Silos Metálicos" en la CCS-F "Fidel Claro".

Proyecto "Bambú" CCS-F "Fidel Claro".

Promoción de la equidad de género en el sector cooperativo y campesino del municipio Camajuaní, Villa Clara, en un marco de avance hacia la soberanía alimentaria.

Disminución de las emisiones de metano a la atmósfera mediante el uso de biodigestores tubulares de polietileno en el manejo de estiércol de ganado porcino (seleccionados 15 productores, 2 mujeres).

Introducción de las fuentes renovables de energía en la comunidad "Las Lechugas".

Movimiento Agroecológico: "Campesino a Campesino".

La ACTAF y el CUM también conducen los proyectos:

Fortalecimiento del Sistema de Extensión Agraria para la Agricultura Urbana de Villa Clara.

Proyecto de Capacitación de los Técnicos de Base. ACPA no tiene proyectos con financiamiento en Camajuaní. El MINAGRI en Camajuaní no desarrolla ningún proyecto de Iniciativa de Desarrollo Local endógeno del municipio y que sea sustentado con los recursos internos sin buscar financiamiento de organizaciones en el exterior.

1. Proyecto \# 1: Fomento de la producción de Palma Real para la reforestación:

En la CCS Arnaldo Arteaga de La Sabana existe experiencia y tradición en cultivo y propagación de la palma real que diversifica las opciones para poblar grandes áreas deforestadas. El proyecto pretende crear condiciones para la producción de la palma real, rescatando una planta endémica de la zona que se empleará fundamentalmente en la reforestación. Contribuye a la repoblación forestal mediante la siembra y propagación de la palma real con beneficios como aprovechar sus frutos para alimento animal y usar hojas y tallos para diversas construcciones, lográndose plantar más de 5000 palmas. Se creó nueva infraestructura de viveros que generan nuevos empleos y aportan rendimiento económico a la CCS por venta de posturas a otras cooperativas, fortaleciendo la actividad económica en las organizaciones de base.

Aumenta la presencia de la mujer en el sector agrícola al abrir 3 empleos destinados a mujeres. Adquiere un tractor que reporta beneficios para la cooperativa y el poblado como medio de transporte. Se aplican técnicas agroecológicas para cultivar la palma real, ahorra fertilizantes y productos químicos, mejora el medio ambiente y protege la salud humana, aprovecha los desechos de cosechas y abonos naturales que mantienen en equilibrio el ecosistema y garantizan una agricultura ecológica. Introduce nuevas técnicas para la producción de la palma real y diversifica la producción intercalando con la palma real otros cultivos como el plátano.

Se fortalece el capital humano a través de capacitación a nuevos productores y a las mujeres aportando nuevos conocimientos sobre el cultivo de la palma real y las prácticas agroecológicas, según plantea Alhama (2008). También se logra incorporar instituciones como las escuelas donde crearon dos círculos de interés para la enseñanza primaria.

\section{Proyecto \# 2: Fabricación de Silos Metálicos en la CCS Fidel Claro.}

Se inició en diciembre de 2008 con el objetivo de elevar la conservación de granos básicos, elevar el nivel de vida de productores y familiares y ayudar a la seguridad alimentaria. Financiado por la Agencia Suiza para el Desarrollo (COSUDE) que aportó parte considerable del financiamiento en moneda libremente convertible y la ANAP que aportó, por la parte cubana, en moneda nacional.

La pérdida de granos con la utilización de métodos tradicionales de conservación era, según series históricas, $30 \%$ a 50\% en maíz, 20 a $25 \%$ en frijol, $20 \%$ en arroz, y 50\% y más en sorgo. Eso demuestra la relevancia de esta nueva tecnología y el crecimiento del suministro de estos productos en el mercado interno.

El Proyecto está vigente solo en la cooperativa objeto de estudio. Cuenta con un productor de silos, iniciador del proyecto que posee muchos conocimientos sobre la fabricación, uso, manejo y conservación de los silos, lo cual le ha permitido capacitar a diferentes productores en el país. Al cierre del año 2015 tenía una producción acumulada de 1412 silos lo que representa un ahorro en pérdidas de cosechas de 39110.4 qq. Las capacidades de los silos se proyectan a las necesidades del comprador: 1 qq. con precio $\$ 116,83,2$ qq. con precio $\$ 163.18,4$ qq. con precio $\$ 339,36,8$ qq. con precio $\$ 290.00,12$ qq. con precio $\$ 370.00,18$ qq. cuesta $\$ 522.68$ y 30 qq. cuesta $\$ 800.00$.

La fabricación de silos metálicos creó una nueva fuente de empleo e ingresos a la cooperativa y beneficia directamente a los familiares de los productores. Brinda a la cooperativa una fuente de ingreso adicional, con la venta de los silos y venta de las producciones marginales o secundarias que puedan generarse de los residuos de su fabricación como cubos, regaderas, recogedores y otros utensilios demandados por la comunidad. Los silos ayudan a aprovechar el espacio, mejora de condiciones higiénicas en la vivienda del productor y otros locales cercanos a su finca y hace más agradable y sana la vida de las familias. Ayuda a las mujeres que deben manipular sistemáticamente los granos al preparar los alimentos para la familia y la cría y engorde de animales de corral. Con la utilización de los silos se preserva el $100 \%$ de los granos con buena calidad, garantizando la seguridad alimentaria sin uso de productos químicos. 
3. Proyecto \# 3: Reforestación con bambú como alternativa ecológica en la producción sostenible de materiales de construcción y viviendas, en la CCS Fidel Claro:

Desde Julio de 2012 se ejecutó financiado por COSUDE con el objetivo de crear la base para desarrollar una economía local de bambú en condiciones cubanas, que resulte atractiva como forma de desarrollo para comunidades rurales y pudiera ser replicada en Cuba y Latinoamérica. Las líneas principales del proyecto son: (a) propagar y sembrar varias especies de bambú que se adapten a las condiciones cubanas, (b) implementar formas de procesamiento y empleo del bambú en producción de artículos de artesanías y madera prensada, y (c) producir materiales de construcción y construir viviendas usando bambú.

Se concretó la introducción de una nueva variedad de bambú para repoblación forestal en las riberas de ríos dónde tiene funciones de conservación de suelos y mitigación de desastres. Su cultivo es rentable a corto plazo y han construido viveros de posturas para garantizar continua reforestación. Hasta el momento se construyó el taller y hay materia prima y maquinarias para producir muebles, útiles del hogar y elementos decorativos de gran demanda, con mercados seguros. El proyecto genera a la CCS una fuente de ingreso adicional y es una contribución económica a nivel macro, pues genera nuevos empleos. Trabajan 3 hombres y 8 mujeres con un enfoque de género, se rescatan oficios como carpintería, artesanía y trabajo agrícola y desarrolla talentos locales. Aunque el proyecto trabaja con la línea fundamental del bambú, en el taller se dan servicios de aserrado de madera y produce posturas de frutales y forestales para el desarrollo agrícola de la localidad.

4. Proyecto \# 4: Promoción de equidad de género en el sector cooperativo y campesino del municipio Camajuaní:

Desarrollado en 5 cooperativas: CCS Benito Ramírez, Juan Verdecia y Miguel A. Acevedo y CPA Niceto Pérez y Juan Bautista Montes de Oca. Tiene el objetivo de aplicar medidas que favorezcan la equidad en las relaciones de género en el sector campesino, en un marco de avance hacia la soberanía alimentaria y mejora de las condiciones de vida. Cuenta con 155364.06 euros, aportados por la ONG Mugarik Gabe y CUP $\$ 71120.00$ aportados por la ANAP.

Generó nuevos empleos en mayoría para mujeres y, en algunos casos, ubica hombres y mujeres en trabajos no tradicionales. Realiza acciones formativas técnicas, gerenciales y de empoderamiento para mujeres, formación y concientización en equidad de género para cooperativistas, campesinado y directivos de cooperativas y capacitación técnica agropecuaria. Dotó a las cooperativas de infraestructura y equipo para producción agroecológica. Creó instalaciones que alivian el trabajo doméstico a la mujer y generan empleo como casa para el cuidado de niños por vías no formales y lavandería. Logró diversificar la producción de alimentos en las cooperativas, especialmente frutas, vegetales y alimentos en conservas y proteicos con el objetivo de procesar frutas, hortalizas y condimentos y su conservación por métodos artesanales. Minimizó los picos de cosecha, aumentando el volumen y disponibilidad de alimentos todo el año y diversificando las opciones de cocina doméstica para la familia. Ello propicia mejor nutrición y beneficio económico a los cooperativistas. Logró eficiencia y rentabilidad de la actividad agropecuaria con soluciones sostenibles $\mathrm{y}$ adaptables ante las condiciones actuales y tendencias futuras, con mayor producción de carne, leche y huevos a partir de la infraestructura que creó en cada cooperativa.

CCS Benito Ramírez: El nuevo centro de elaboración de alimentos asegura disponibilidad y diversidad de frutas, vegetales, hortalizas y condimentos todo el año a partir de frutas y vegetales que se pierden en picos de cosecha (30 qq. anuales). Con la reforestación y mejoramiento de condiciones de trabajo rural incrementa los rendimientos, diversifica la producción y crean conservas de puré y pasta de tomate, mermeladas, encurtidos, vinagre, jugos y siropes.

CCS Juan Verdecia: Además de tabaco, tiene producciones estables de viandas y hortalizas que pueden duplicarse con la mejora de la infraestructura productiva alcanzada con el proyecto.

CCS Miguel A. Acevedo: Las acciones concebidas permiten duplicar y diversificar los cultivos, con el montaje de la casa de posturas, la cual proporciona ganancias con la venta de semillas.

CPA Niceto Pérez: Se prevé instalar la corriente eléctrica para incrementar el área bajo riego en 13.42 ha. Ello traerá consigo el incremento de los rendimientos de los cultivos y su diversificación.

CPA Juan Bautista Montes de Oca: Construyó nuevas instalaciones para animales, reparó la vaquería e incrementó 3 ha. de pastos para triplicar la producción de leche y carne. Introdujo la producción de leche de cabra y huevos.

5. Proyecto \# 5: Disminución de emisiones de metano al aire con uso de biodigestores en el manejo de estiércol porcino

Invirtió 33 528.00 CUC del PNUD, PPD y FMAM1 y CUP $\$ 13411.00$, destinados a 15 actores, 13 hombres y 2 mujeres. Es demostrativo para disminuir emisiones de metano y óxido nitroso con introducción de los digestores tubulares de polietileno en tratamiento anaeróbico de excretas de cerdos en 9 fincas y 3 áreas de cooperativas, ubicadas en el frágil ecosistema costero norte de Villa Clara. Instaló recursos y creó capacidades que mejoran la calidad de vida campesina.

Jorge Valeriano, con 10 cerdos, Omar Martínez que tiene 20, José A. Martín con 30 cerdos, Pablo Martín con 100 y María del Carmen Carvajal con 120, suman 280 cerdos que representan el $38 \%$ de los involucrados a nivel municipal. Logran reducir con los biodigestores en $413.71 \mathrm{~kg}$. la emisión de metano a la atmósfera en la cooperativa. Utilizan el metano del biodigestor como fuente de energía alternativa para cocer alimentos y alumbrado y reducen el uso de leña para cocinar. Mejora la salud familiar al reducir los gases tóxicos de la combustión incompleta de leña. Fertilizan el 
suelo con efluentes de la digestión anaeróbica lo que benefició 120 ha., sembradas con viandas, hortalizas, granos y frutales.

Empoderan a campesinos y campesinas con nuevas tecnologías y conocimientos a través de la capacitación para construir, manejar y operar la tecnología de biodigestores, el uso integral del gas y los efluentes. Reducen la emisión de metano y óxido nitroso del manejo de estiércol porcino líquido que superaban $1000 \mathrm{~kg}$./año. Al bajar el consumo de leña redujeron la deforestación y conservan la biomasa boscosa. La tecnología produce biofertilizante rico en nitrógeno, fósforo y potasio capaz de competir con fertilizantes químicos, caros y que afectan al medio ambiente.

6. Proyecto \# 6: Introducción de fuentes renovables de energía en la comunidad Las Lechugas de la CCS Fidel Claro:

Financiado por el PNUD, PPD y FMAM que donó 46109.00 CUC, con \$13 872.00 en moneda nacional. Introdujo el uso de fuentes alternativas de generar energía fotovoltaica como fuente renovable para electrificar el poblado y el bombeo eólico en suministro de agua. Trabaja la reforestación con especies forestales y frutales y el control de plantas invasoras. Disminuye el uso de combustibles fósiles y leña con introducción de cocinas ahorradoras que mejoran la calidad de vida de la familia evitando la exposición a gases contaminantes. Frena la deforestación y destrucción del hábitat natural de muchas especies. Contribuye a restaurar y conservar la biodiversidad en su entorno a través del fomento de especies forestales y frutales al fomentar un vivero comunitario con capacidad de 10000 posturas anuales para la reforestación.

Beneficia a campesinos de la cooperativa y de la CCS José Martí. Instaló paneles solares para electrificación. El sistema de bombeo de agua tiene mangueras, tanques y paneles solares comprados por el proyecto. Construyó un vivero, adjunto al taller de bambú, con sistema de bombeo para regar las posturas. Aumenta la productividad y uso racional de los recursos naturales con un programa de capacitación comunitaria en educación ambiental, empleo de nuevas tecnologías de celdas fotovoltaicas y cocinas eficientes y prácticas de agricultura ecológica.

\section{Proyecto \# 7: Movimiento Agroecológico Campesino a Campesino:}

Ha sido dirigido por la ANAP como organización líder del campesinado. Se orienta a promover prácticas agroecológicas en los sistemas agrarios, no solo de cultivo, sino también en la ganadería, reforestación y conservación de los ecosistemas agrícolas. Surgió en la provincia de Villa Clara en 1997, auspiciado por la organización no gubernamental Pan para el Mundo y su objetivo como movimiento agroecológico es rehabilitar más del 50\% de las tierras cultivadas y que más del $50 \%$ de los campesinos en cooperativas involucradas sean promotores.
Camajuaní fue desde el inicio abanderado de este movimiento agroecológico y del proyecto que cambió la forma de pensar de los campesinos. Actualmente el municipio tiene 48 facilitadores, 40 hombres y 8 mujeres y 95 promotores, 87 hombres y 8 mujeres. Se pretende que cada campesino del municipio sea promotor de las prácticas agroecológicas. Se fabrica abono orgánico o compost mediante 150 lombriculturas que alcanzaron una producción acumulada hasta marzo de 2016 de 1046 t. de humus. 3643 productores de materia orgánica tienen una producción acumulada de $9156 \mathrm{t}$. Se han categorizado 64 fincas en: Categoría 1 Iniciando el camino agroecológico (37), Categoría 2 Finca en transformación agroecológica (27) y Categoría 3: Finca agroecológica (1). Se diversifica y aumenta la producción por la vía de incrementar rendimientos con menor uso de agroquímicos. El campesino se apodera de nuevas formas de producción aprovechando los recursos de su finca y optando por producciones ecológicas para lograr una agricultura sana, con mayor interacción entre cooperativas y fortalecimiento de relaciones entre cooperativistas pues la esencia del proyecto es que el campesino promueva esas prácticas y las trasmita a otros cooperativistas y nuevas generaciones.

8. Proyecto \# 8: Fortalecimiento del Sistema de Extensión Agraria para la Agricultura Urbana de Villa Clara.

Financiado por ACDI Canadá que aportó $\$ 95000$ y la ACTAF \$177 507 con el objetivo de contribuir al desarrollo de modelos socioeconómicos sostenibles para producciones urbanas con participación local y manejo racional del medio ambiente. Tiene como esferas de interés prioritarias un ambiente institucional facilitador, el desarrollo económico local, la oferta de servicios públicos comunitarios y la sostenibilidad ambiental a nivel de la comunidad. Contribuye al desarrollo del movimiento de Agricultura Urbana en el municipio a través del fortalecimiento del Sistema de Extensión Agraria y la adopción de prácticas agrícolas sostenibles y respetuosas del medio ambiente. Creó una Red de información científico-técnica que facilita conocimiento a extensionistas, técnicos y productores. Dotó a decisores, productores y técnicos de capacidad de gestión del conocimiento necesario para lograr producciones eficientes, prevenir riesgos provocados por desastres naturales y lograr equidad de género. Fueron reconocidos y estimulados los mejores 8 productores y productoras que integran las prácticas agroecológicas complementando sus módulos de producción. Aumentó la capacidad tecnológica y creó una mejor infraestructura local.

\section{Situación de los proyectos en laCCS "Fidel Claro"}

Como se puede observar la cooperativa objeto de estudio se desarrolla sobre bases económicas y productivas fuertes. Es capaz de enfrentar cualquier reto que signifique beneficios tanto para ella como para sus cooperativistas, los cuales tienten la disposición y la voluntad de enfrentar esos retos junto con el aparato administrativo de la cooperativa. Hoy la CCS está inmersa en 4 proyectos y el movimiento agroecológico. 
En el movimiento agroecológico la cooperativa cuenta hoy con 10 promotores, todos hombres, 2 facilitadores una mujer y un hombre, 6 lombriculturas para la producción de humus de lombriz y 10 fincas categorizadas como Fincas en Transformación Agroecológica (categoría 2) que representan el $37 \%$ de las fincas en esa categoría del municipio.

El Proyecto Post-Cosecha: "Fabricación de Silos Metálicos" está vigente solo en la cooperativa. Cuenta con un productor de silos, el cual fue iniciador del proyecto y posee muchos conocimientos sobre la fabricación, uso, manejo y conservación de los silos, lo cual le ha permitido ofrecer capacitación a diferentes productores en el país. Al cierre del año 2010 cuenta con una producción acumulada de 412 silos lo que representa un ahorro en pérdidas de cosechas de 9110.4 qq.

El Proyecto "Bambú" está siendo aplicado solamente en esta cooperativa del municipio. Cuenta con un taller instalado que tiene toda la maquinaria e implementos de trabajo necesarios para la producción, pero aún no cuenta con la licencia de comercialización para la venta de sus producciones. Además cuenta con un vivero que tampoco ha empezado a producir pero se espera la producción de posturas de frutales y maderables para la reforestación, la venta a la población y como futura fuente de materia prima.

El Proyecto "Disminución de las emisiones de metano a la atmósfera mediante el uso de biodigestores tubulares de polietileno en el manejo de estiércol de ganado porcino" incluye a 5 productores de la CCS los cuales están interesados en la instalación de los biodigestores y el disfrute de sus beneficios. Estos son Jorge Valeriano que cuenta con 10 cerdos, Omar Martínez que tiene 20, José A. Martín con 30 cerdos, Pablo Martín con 100 y María del Carmen Carvajal que cuenta con 120 cerdos, para sumar un total de 280 cerdos que representan el $38 \%$ de los involucrados a nivel municipal. Logran reducir con la instalación de los biodigestores en $413.71 \mathrm{~kg}$. las emisiones de metano a la atmósfera en la cooperativa.

El Proyecto "Introducción de las fuentes renovables de energía en la comunidad "Las Lechugas", beneficia a campesinos asociados a la cooperativa y también a los de la CCS José Martí. Cuenta con la instalación de los paneles solares para su electrificación beneficio del cual actualmente ya gozan. El sistema de bombeo de agua no se ha terminado aún pero los materiales están comprados disponibles como son las mangueras, los tanques y los paneles solares. También se espera la construcción de un vivero que será una dependencia del creado en el taller de bambú pero este no puede ser materializado hasta tanto no se termine la instalación del sistema de bombeo para así lograr un correcto riego de las posturas a sembrar.
E. Principales problemas de sostenibilidad de los proyectos agropecuarios en Camajuaní.

1. Sociales y culturales

Se ajusta el movimiento agroecológico y el proyecto de los biodigestores pues no existe cultura medioambiental ni conocimientos suficientes acerca de la agricultura sostenible por lo que en ocasiones se hace muy difícil promover prácticas agroecológicas para la producción de cultivos y el funcionamiento general de una finca. Hay campesinos arraigados a la agricultura convencional que no consideran cambiar su forma de producir.

\section{Económicos}

La dificultad inicial era que las cooperativas no tenían cuenta en divisas para poder comprar materiales y recursos productivos que garantizaran la sostenibilidad de los proyectos. Por ejemplo; el proyecto de fabricación de silos hace algún tiempo estuvo casi a punto de desaparecer pues terminó el financiamiento y no se contaba con la divisa para poder comprar las planchas de zinc hasta que AZCUBA se interesó por el mismo y decidió ser el proveedor de la materia prima pues esta entidad tiene acceso a una cuenta en divisa. El otro proyecto que puede verse afectado es el del taller de la fabricación de útiles y muebles de bambú, en la adquisición de piezas de repuesto.

El Proyecto Las Lechugas es una obra social con enfoque medioambientalista pero depende de los pobladores de la zona que sea sostenible en el tiempo, mediante el manejo y cuidado adecuado de los recursos a su disposición y con la concientización de todo el poblado al cuidado de la flora y fauna en la zona que está afectada por la tala indiscriminada.

\section{F. Beneficios de los proyectos de desarrollo}

Agropecuario local: Los proyectos inciden positivamente en la situación de las cooperativas e irradian positivamente en la estructura productiva del municipio. En las cooperativas se pueden generar empleos con incorporación de la mujer en actividades agrícolas como el trabajo en los viveros, composteo, lombricultura, conservación de alimentos y dirección de procesos. Otra fuente de empleo es el taller de bambú que además del vivero tiene otras actividades como la fabricación de artesanías, el aserrado y la producción de muebles que requieren de mano de obra.

El taller de bambú genera desarrollo a la localidad y municipio. Tiene amplias perspectivas de comercialización de sus productos en cadenas mayoristas y minoristas y creación de una sala expositiva dentro del centro para atraer a turistas y futuros clientes hacia sus productos. Esto genera un remanente en divisas y da paso a un cambio en el aparato económico y financiero de la cooperativa, logrando tener una cuenta bancaria en divisas. 
Otro factor que se evidencia positivamente es la diversificación de las producciones pues los promotores de prácticas agroecológicas cuentan con fincas con producciones variadas, patios de referencia nacional con una gama de frutales, plantas medicinales y ornamentales. Ello contribuye a la repoblación forestal y al cuidado de la flora y la fauna, creación de insumos productivos como abono orgánico para fertilizar los suelos y piensos para la alimentación de los animales.

Se reportan beneficios económicos con la fabricación de silos pues representa una fuente de ingresos para la cooperativa y el país. Se ahorra por salvar producciones pues el silo constituye un envase seguro y ecológico para las producciones conservando el $100 \%$ de los granos que sean almacenados. Es una inversión duradera que reporta beneficios y es más factible su compra que la de otros medios de almacenamiento como sacos que escasean, no tienen durabilidad y las plagas y animales pueden atacar los productos con mayor facilidad. También reportan beneficio a la salud humana con un control de vectores y plagas que atraen enfermedades perjudiciales a las personas.

Otros beneficios a la salud humana los reportan la instalación de biodigestores que logran disminución de más del $95 \%$ de las emisiones de metano al medio. Sustituyen la cocina de leña por una cocina más eficiente que trabaja con gas del biodigestor, con disminución de enfermedades respiratorias agudas. Se reportan beneficios al medio ambiente con la utilización de técnicas agroecológicas, mediante acciones de repoblación forestal de agentes contaminantes.

Estas cooperativas son ejemplo de cómo los proyectos son promotores del desarrollo y contribuyen al fortalecimiento del tejido productivo agrícola a través de acciones como la creación de nuevas infraestructuras, generación de empleos con enfoque de género, generación de fuentes de ingresos, fabricación artesanal de piensos e insumos y repoblación forestal. Se aumentan los resultados productivos por la vía del incremento de los rendimientos con menor uso de agroquímicos. Se fomenta la conservación de semillas en la localidad, uso de fuentes de energía alternativas como la solar y el biogás y diversificación de las producciones introduciendo nuevos productos como puré de tomate y ají, pulpas y jugos de frutas, dulces secos y en almíbares, vinagres, vinos, condimentos y conservas.

\section{Trabajos futuros}

Este estudio generó una nueva línea de trabajo que dio lugar a un Proyecto de Investigación del Ministerio de Educación Superior para modelar las funciones y roles de cada uno de los actores locales en función del Desarrollo Agropecuario Local en un Municipio Cubano. Este Proyecto tributará al trabajo de Tesis Doctoral en Ciencias de la Educación del primer autor, profesor del CUM Camajuaní, a realizarse con la colaboración del Centro de Estudios de Educación Superior de la Universidad Central Marta Abreu de Las Villas.

\section{Concluciones}

Los proyectos actúan como dinamizadores del tejido productivo local y proporcionan acciones de cooperación entre actores locales.

El sector agrícola está estructurado en formas de propiedad social, cooperativa y privada donde el sector cooperativo y campesino es el principal productor aportando más del 95\% de la producción.

Los proyectos contribuyen al fortalecimiento del tejido productivo agrícola de Camajuaní en aspectos tecnológicos, técnicos, productivos, organizacionales, sociales, medioambientales y otros.

La consolidación de los tejidos productivos tiene como soporte de funcionamiento el entramado institucional que tiene gran importancia en la promoción del desarrollo económico local.

Los proyectos contribuyen al Desarrollo Local con la generación de empleos, la incorporación de la mujer al trabajo agrícola, incrementando la repoblación forestal, disminuyendo las emisiones de gases, salvando especies en peligro y protegiendo los agroecosistemas.

\section{Agradecimientos}

Los autores reconocen las contribuciones del MSc. Israel Alonso Díaz, Especialista Municipal delhttps://es.overleaf.com/project CITMA en Camajuaní, de la socióloga Annia Martínez Massip y de los Funcionarios del Gobierno local, la Delegación de la Agricultura, la ANAP, la ACTAF, los directivos de las Cooperativas campesinas y los profesores y estudiantes del CUM Camajuaní en la realización del trabajo y por sus criterios técnicos y apoyo en la elaboración de este artículo.

\section{Referencias}

[1] C. Hernández, "Utilización del trabajo por proyectos para incentivar la innovación tecnológica en los estudiantes universitarios," Revista Científica de la Fundación Iberoamericana para la Excelencia Educativa Hecademus, vol. 3, no. 8, pp. 42-54, 2010.

[2] M. Lloret and E. Méndez, "Planificación del desarrollo territorial y local en cuba, aspectos conceptuales, metodológicos, y estratégicos," Facultad de Ciencias Económicas. Universidad Central "Marta Abreu" de Las Villas, 2007.

[3] R. Flores and D. Figueras, "Papel de la gestión de proyectos en el fortalecimiento del tejido productivo agrícola de camajuaní," Fac. Ciencias Económicas. Departamento de Economía. Universidad Central Marta Abreu de Las Villas, 2001.

[4] J. Koch, "Manual del empresario exitoso," EUMED. Edición electrónica, disponible en: http://www.eumed. net/libros/2006c/210, 2006. 
[5] C. Hernández, K. Faye, and E. Cárdenas, "Educación superior cubana y políticas de desarrollo rural sostenible: Rol del diagnóstico sociocultural y el programa de desarrollo local en el desarrollo de las comunidades rurales en cuba," LAP LAMBERT Acad. disponible en: http://www.amazon. com/Educaci\%C3\%B3n-Superior-Pol\%C3\%

ADticas-Desarrollo-Sostenible/dp/3847360671/ref= sr_1_7/190-1540178-4824760?s=books\&ie=UTF8\& qid $=1383333871 \& s r=1-7,2011$.

[6] L. Fajardo, D. Figueras, and Y. Roque, "Algunas consideraciones sobre el trabajo por proyectos y el desarrollo humano local en espacios rurales," Universidad Central “Marta Abreu” de Las Villas, 2008.

[7] C. A. H. Medina, M. A. Carrasco, R. Garcés González, and R. Casas, "Colaboración y alianzas del cum a la red local de conocimiento e innovación: estudio de caso de camajuaní, cuba," p. 111, 2016.

[8] C. A. H. Medina and M. A. Carrasco, "Integración de proyectos agropecuarios al desarrollo local. caso de estudio del centro universitario municipal camajuaní," GUCID, no. 54, pp. 1-11, 2015.

[9] C. A. H. Medina and E. Cárdenas, "Sistema de acciones para mejorar la cultura ambiental y la calidad de vida de la comunidad suburbana de arroyo frío, camajuaní, cuba," MONFRAGÜE Desarrollo Resilente, vol. 4, no. 2, pp. 134-153, 2015.

[10] C. A. Hernández Medina, "Trabajo por proyectos con estudiantes de agronomía para activar la innovación tecnológica en la nueva universidad cubana," Revista de Ciencia y Tecnología, no. 23, pp. 4-12, 2015.

[11] A. Báez Hernández, C. A. Hernández Medina, J. M. Perdomo Vázquez, R. Garcés González, and M. Alibet Carrasco, "Modelo de gestión del conocimiento para el desarrollo agropecuario local," Estudios sociales (Hermosillo, Son.), vol. 28, no. 51, pp. 2-26, 2018.

[12] C. A. H. Medina, J. M. P. Vázquez, R. G. González, and M. A. C. Fuentes, "Gestión universitaria del conocimiento para el desarrollo local," Lámpsakos, no. 18, pp. 44-57, 2017.

[13] A. B. Hernández, C. A. H. Medina, J. M. P. Vázquez, R. G. González, and M. A. C. Fuentes, "Praxis de una red local gucid y desarrollo local," Revista Ingeniería Industrial, vol. 18, no. 3, pp. 253-266, 2018.

[14] C. A. Hernández, "Roles de los centros universitarios municipales (cum) en el desarrollo local de los municipios cubanos," Ingenierías USBMed, vol. 3, no. 1, pp. 43-49, 2012. 